\title{
The effect of air exposure on device performance of flexible C8-BTBT organic thin-film transistors with hygroscopic insulators
}

\author{
Pengshan Xie, Tianjiao Liu, Pei He, Guozhang Dai, Jie Jiang, Jia Sun ${ }^{*}$ and Junliang Yang*
}

\begin{abstract}
Organic thin film transistors (OTFTs) are normally sensitive to ambient conditions and show performance degradation in air. On the contrary, the performance of flexible 2,7-dioctyl[1] benzothieno [3,2-b][1]benzothiophene (C8-BTBT) OTFTs using cross-linked polymer layer, poly(4vinyl-phenol)-4,4'-(hexafluoroisopropylidene) diphthalic anhydride (PVP-HDA), as the dielectric layer can be improved in air conditions with $\mathbf{4 0} \%$ relative humidity. Under soaking in air with $\mathbf{4 0} \%$ relative humidity, the electrical behavior, surface morphology, and contact angle of the flexible C8-BTBT OTFTs using PVP-HAD as dielectric layer with three different thicknesses were investigated. It is found that, when the devices with $375 \mathrm{~nm}$-thick PVP-HDA films are placed in $40 \%$ relative humidity air conditions for $6 \mathrm{~h}$, the corrected average mobility $(\mu)$ can increase from 3.2 to $5.1 \mathrm{~cm}^{2} \mathrm{~V}^{-1} \mathrm{~s}^{-1}$. Furthermore, the average threshold voltage $\left(V_{\text {th }}\right)$ changes from -12.4 to $-9.3 \mathrm{~V}$ while keeping a constant ratio of $I_{\mathrm{on}} / I_{\text {off }}=10^{4}$. These results indicate that the flexible C8-BTBT OTFTs with PVP-HDA dielectric layer exhibit interesting application prospects.
\end{abstract}

Keywords: flexible electronics, C8-BTBT, OTFTs, PVP-HDA, hygroscopicity, air stability.

\section{INTRODUCTION}

Over the past years, significant developments in organic thin film transistors (OTFTs) have been made, and the mobility $(\mu)$ has been increased to over $43 \mathrm{~cm}^{2} \mathrm{~V}^{-1} \mathrm{~s}^{-1}[1]$. It is well known that OTFTs have the advantages of solution process, flexibility, low cost, large area, and so on [2-5]. Thus, OTFTs show great potential applications in sensors, circuits, and synaptic electronics [6-11], also thanks to the improvement of device performance via new active materials, device engineering, interface en- gineering of dielectric layer, etc. [12-16]. Air stability is one of the most important issues for meeting the requirements in practical applications [17-21]. In previous studies, organic materials affected by air environment have been widely studied $[22,23]$. It has been reported that for gate dielectric materials with active functional groups, such as hydroxyl groups, the interaction with the adsorbed water in air would affect the device performance $[24,25]$, leading to performance degradation in air $[26,27]$.

As a typical gate dielectric layer material, cross-linked polymer, poly(4-vinyl-phenol)-4,4'-(hexafluoroisopropylidene) diphthalic anhydride (PVP-HDA), can be prepared via solution processes and exhibits excellent insulating properties [28]. Furthermore, 2,7-dioctyl[1] benzothieno[3,2-b][1]-benzothiophene (C8-BTBT) is one of the most promising high-mobility $\mathrm{p}$-channel semiconductor material in OTFTs [29-31]. Herein, the effect of air exposure with $40 \%$ relative humidity on the device performance of flexible C8-BTBT OTFTs with crosslinked polymer PVP-HDA as the dielectric layer was studied in details. The flexible C8-BTBT OTFTs were fabricated using a structure of bottom-gate/top-contact on poly(ethylene terephthalate) (PET) covered with indium tin oxide (ITO) as the gate electrode. The mobility of the device was found to increase initially and then decline upon increasing the exposure time. Since the C8BTBT has good air stability and the PVP-HDA crosslinked polymer gate dielectric film has the ability to absorb water from air, it is deduced that the performance changes are mainly caused by the PVP-HDA films. By comparing devices consisting of PVP-HDA films with different thicknesses, namely 650, 375, and $280 \mathrm{~nm}$, it can be seen that the humidity influence becomes weaker with

Hunan Key Laboratory for Super-microstructure and Ultrafast Process, School of Physics and Electronics, Central South University, Changsha 410083, China

* Corresponding authors (emails: jiasun@csu.edu.cn (Sun J); junliang.yang@csu.edu.cn (Yang J)) 
decreasing thickness of PVP-HDA films.

\section{EXPERIMENTAL SECTION}

C8-BTBT was purchased from Jilin Optical and Electronic Materials Co., Ltd. and used without purification. PVP, HDA, and triethylamine (TEA) were purchased from Sigma-Aldrich, and were also employed without further purification. OTFTs were fabricated with the conventional structure, consisting of bottom-gate/topcontact. The flexible PET films covered with ITO were selected as substrates. Furthermore, the substrates were ultrasonically cleaned with DI water, acetone, and isopropanol for $5 \mathrm{~min}$ each. After cleaning, the films were dried with nitrogen gas. PVP $(1 \mathrm{~g})$ and $100 \mathrm{mg}$ of HDA were dissolved in $5 \mathrm{~mL}$ of propylene glycol monomethyl ether acetate (PGMEA), separately. In order to achieve a better dissolution, the two solutions were stirred overnight. Afterwards, $10 \mu \mathrm{L}$ of TEA was added into the PVP solution in PGMEA and mixed thoroughly. PVP and HDA solutions were then mixed and stirred for $2 \mathrm{~h}$ to obtain a full cross-link between the two materials. Finally, the solution was filtered through a $0.45-\mu \mathrm{m}$ syringe filter. Central spin coating (CSC) was used to fabricate the gate dielectric layer. During the CSC deposition, spin-coating speeds of 1000, 3000, and $6000 \mathrm{rpm}$ were adopted to fabricate films with different thicknesses. The PVP-HDA films were placed on the hotplate at $100^{\circ} \mathrm{C}$ for $1 \mathrm{~h}$ to promote the cross-linking reaction after spin coating. During the off-center spin coating (OCSC) process, C8BTBT was dissolved in chlorobenzene (CB) with a concentration of $5 \mathrm{mg} \mathrm{mL}^{-1}$. Polystyrene (PS) was added into the C8-BTBT solution as an additive (10 wt\% of PS) $[1,32]$. The C8-BTBT:PS semiconductor layer was fabricated onto different PVP-HDA films via the same OCSC process in the $\mathrm{N}_{2}$-golve box. Finally, 50 -nm-thick $\mathrm{Au}$ source and drain electrodes were deposited at a pressure of $\sim 8 \times 10^{-4} \mathrm{~Pa}$ on C8-BTBT films with a shadow mask, resulting in a channel width and length of 1000 and $80 \mu \mathrm{m}$, respectively.

The morphologies of the PVP-HDA thin films with different air exposure times and different thicknesses were examined by atomic force microscope (AFM, Agilent Technologies 5500AFM/SPM System). The change of dielectric layer capacitance was measured via an impedance tester (IM3536, HIOKI, Japan). Scanning electron microscope (SEM, FEI Helios Nanolab 600i, USA) was also used to identify the change of oxygen content and display the cross-section morphology of the devices. All OTFTs were measured with a Keithley 4200 SCS semiconductor parameter analyzer under ambient con- ditions with a relative humidity of $40 \%$. The field-effect mobility was extracted from the saturated region of the transfer characteristics according to Equation (1):

$\mu=\frac{2 L}{W} \times \frac{I_{\mathrm{D}}}{C_{\mathrm{i}} \times\left(V_{\mathrm{G}}-V_{\mathrm{th}}\right)^{2}}$,

where $\mu$ the is the field-effect mobility, $W / L$ represents the ratio of channel width/length, $C_{\mathrm{i}}$ is the capacitance per unit area, $V_{\mathrm{G}}$ is the gate voltage, and $V_{\mathrm{th}}$ represents the threshold voltage.

\section{RESULTS AND DISCUSSION}

In order to explore the effects of different exposure times in air with $40 \%$ relative humidity on the device performance, the devices with $375 \mathrm{~nm}$-thick gate dielectric layers were fabricated. The structure of the device is shown in Fig. 1a. The statistical data of $V_{\text {th }}$ and $\mu$ are shown in Fig. 1b, c. The average $\mu$ of these devices increases from 8.1 to $11.5 \mathrm{~cm}^{2} \mathrm{~V}^{-1} \mathrm{~s}^{-1}$ in the first $6 \mathrm{~h}$, and then decreases to 10.4 and $8.7 \mathrm{~cm}^{2} \mathrm{~V}^{-1} \mathrm{~s}^{-1}$ in 12 and $18 \mathrm{~h}$, respectively. Correspondingly, the average value of $V_{\text {th }}$ decreases from -12.3 to $-7.2 \mathrm{~V}$. The $V_{\text {th }}$ drops significantly in the first $12 \mathrm{~h}$ and then gradually saturates within the following $6 \mathrm{~h}$. The photograph of the C8-BTBT flexible OTFT (f-OTFT) array is shown in Fig. 1d. The transfer characteristic curves of the devices with $\mathrm{Si} / \mathrm{SiO}_{2}$ as the substrate and gate electrode are shown in Fig. S1a, and the structure diagram is shown in Fig. S1b. The red curve represents the initial device, while the black curve represents the device after being placed in $40 \%$ humidity air conditions for $18 \mathrm{~h}$. By comparing the two states, it can be seen that there is no big difference in the performance. Therefore, short-term and low-humidity exposure have little effect on the C8-BTBT layer.

At present, there are still several issues concerning the mobility calculation of f-OTFTs. A large number of studies have demonstrated that the OTFT mobility is overestimated when using the traditional transistor theory, since the equations used to calculate the mobility are valid only for ideal OTFTs. Very recently, Choi et al. [33] described several types of error calculations in detail and proposed a parameter, the measurement reliability factor $r$, to quickly gauge the overall effectiveness of mobility.

In order to obtain a more reliable $\mu$ value, the factor $r$ was also introduced in the present work, and it is given by:

$r=\left(\frac{\sqrt{\left|I_{\mathrm{ds}}\right|^{\max }}-\sqrt{\left|I_{\mathrm{ds}}^{0}\right|}}{\mid V_{\mathrm{G}}^{\max }}\right)^{2} \div\left(\frac{W C_{\mathrm{i}}}{2 L} \times \mu\right)_{\text {initial }}$, 

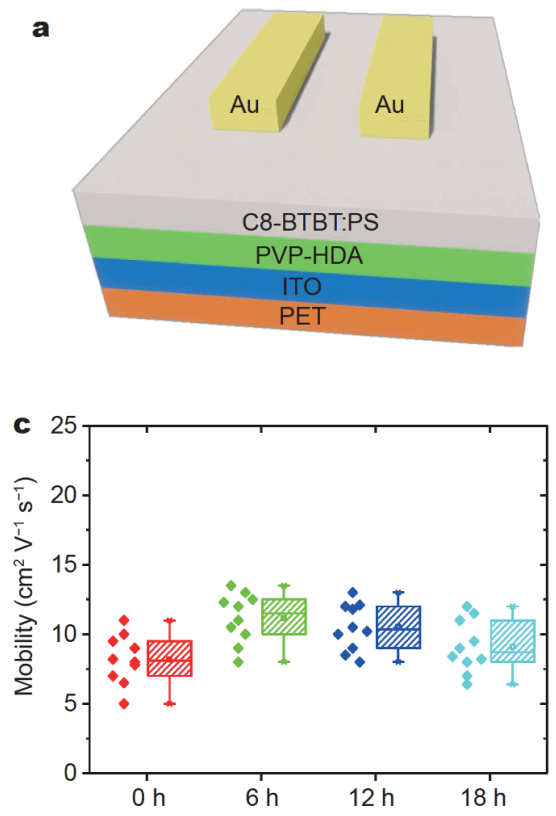

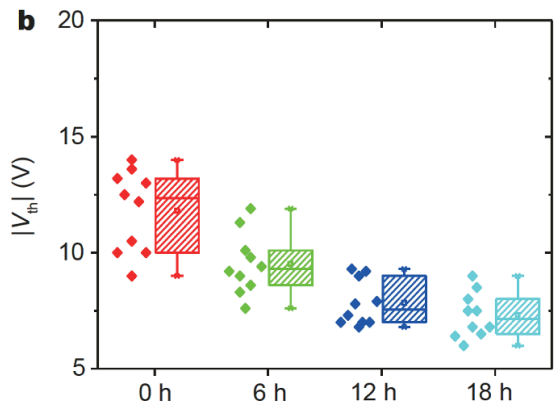

d

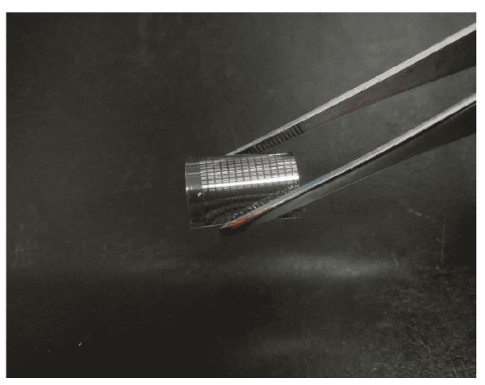

Figure 1 (a) Schematic diagram of the OTFTs with a bottom-gate/top-contact structure. (b) Statistical $V_{\text {th }}$ and (c) $\mu$ of the devices with a 375-nmthick PVP-HDA film. (d) Photograph of the flexible C8-BTBT OTFTs array.

where $\left|I_{\mathrm{ds}}\right|^{\max }$ is the experimental maximum source-drain current at the maximum gate voltage $\left|V_{\mathrm{G}}\right|^{\mathrm{max}}$. According to this method, the mobility of the devices was recalculated as: $\mu_{\text {initial }} \times r$. The transfer characteristic curves of our devices can be considered similar to the one of the overestimated cases described in [33]. Table 1 shows the factor $r$ and corrected $\mu$ of the devices with 375-nm-thick gate dielectric layers.

The typical transfer characteristic curves for the devices with four different exposure times are shown in Fig. 2. The $V_{\text {th }}$ starts to decrease from $-9.5 \mathrm{~V}$ and finally saturates at about $-5 \mathrm{~V}$ (shown in Fig. $2 \mathrm{a}-\mathrm{d}$ ). The $I_{\mathrm{ds}}$ also increases with the exposure time prolonging. However, as the exposure time increases, the $I_{\mathrm{on}} / I_{\text {off }}$ ratio has a significant reduction, as shown in Fig. $2 c$, d. The device exposed for $18 \mathrm{~h}$ shows a decreased $I_{\text {on }} / I_{\text {off }}$ ratio of $10^{2}$. This means that the gate dielectric and the interface between PVP-HDA and C8-BTBT are most subjected to degradation. Furthermore, the hysteresis phenomenon gradually disappears with increasing exposure time, but not in an ordinary way. It is clear that the reduction of the hysteresis window is mainly caused by the obvious reduction of $\left|V_{\text {th }}\right|$, even if the intersection of the reverse sweep extension line and the $X$-axis would increase with longer exposure time. The voltage at the intersection increases from -4 to $-7.8 \mathrm{~V}$. Therefore, the performance of OTFT devices could degrade with exposure to a wet environment, different from the results of many previous
Table 1 The reliable factor $r$ and corrected $\mu$ of the devices with 375-nm-thick gate dielectric layers

\begin{tabular}{ccccc}
\hline Time $(\mathrm{h})$ & 0 & 6 & 12 & 18 \\
\hline$r$ & 0.40 & 0.44 & 0.45 & 0.52 \\
$\mu_{\text {initial }}\left(\mathrm{cm}^{2} \mathrm{~V}^{-1} \mathrm{~s}^{-1}\right)$ & 8.1 & 11.5 & 10.4 & 8.7 \\
$\mu_{\text {corrected }}\left(\mathrm{cm}^{2} \mathrm{~V}^{-1} \mathrm{~s}^{-1}\right)$ & 3.2 & 5.1 & 4.7 & 4.5 \\
\hline
\end{tabular}

reports. The C8-BTBT is a p-type material and has high water-oxygen resistance and air stability [34].

As discussed above, it is speculated that the changes of the performance are related to the PVP-HDA films. It is believed that the PVP-HDA layer could absorb water in air, thus having obvious effects on the performance of the device. In order to further explore the effect of air exposure on PVP-HDA films, the flexible C8-BTBT OTFTs were fabricated with three different thicknesses of dielectric layer.

The characterization of the film thickness was achieved by using a step profiler. Upon increasing the rotation speed, the PVP-HDA films become thinner. The 650, 375, and $280 \mathrm{~nm}$ thicknesses correspond to films fabricated via 1000, 3000, and $6000 \mathrm{rpm}$, respectively. In order to calculate the $\mu$ more accurately, the variation of $C_{\mathrm{i}}$ with thickness and exposure time is shown in Table 2. The specific capacitance extracted from $20 \mathrm{~Hz}$ was used to calculate $\mu$. The $C_{\mathrm{i}}$ would increase with increasing exposure time regardless of the thickness. This also shows 

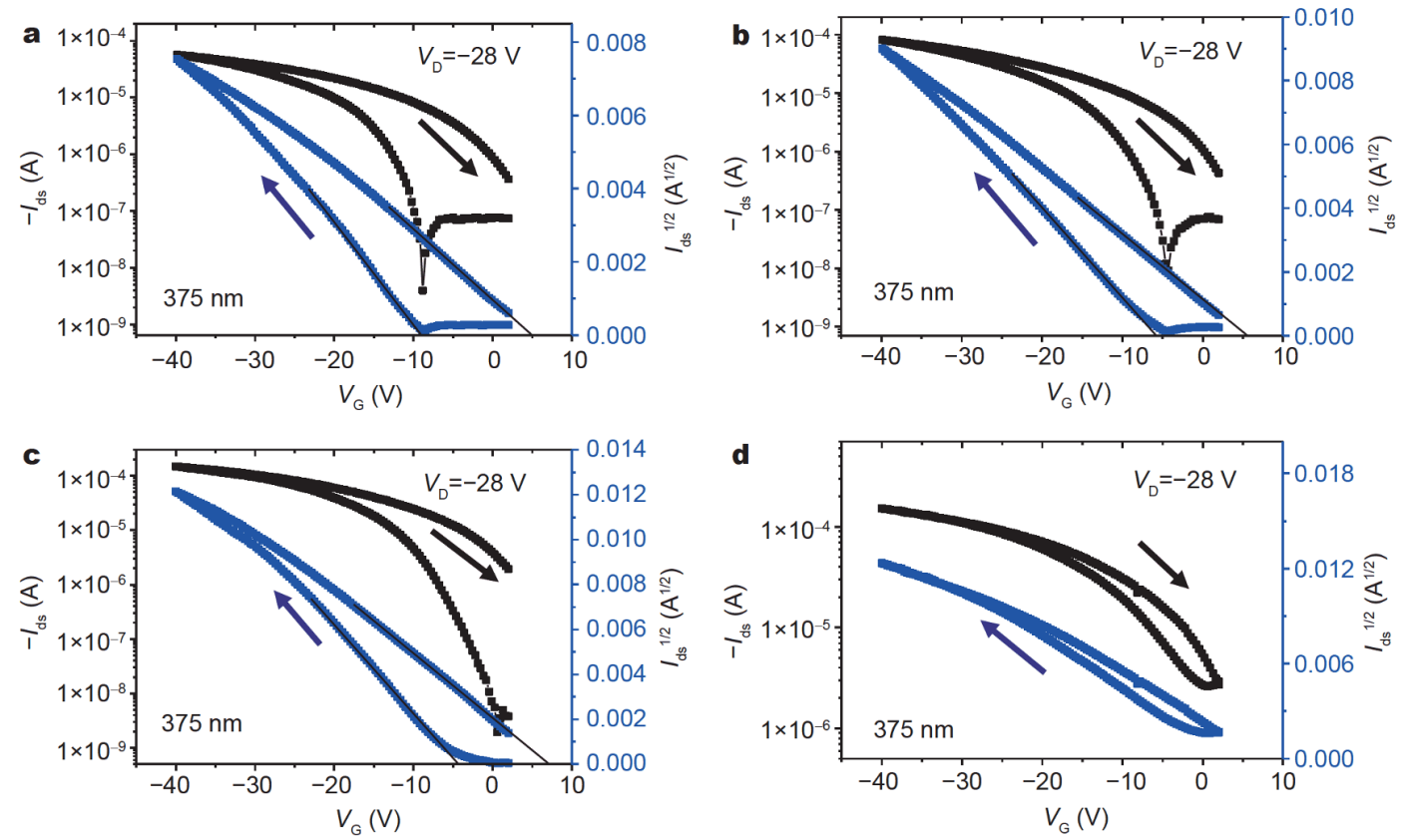

Figure 2 Typical characteristic curves of the device with a 375-nm-thick PVP-HDA film. The device was placed in $40 \%$ humidity air conditions for (a) $0 \mathrm{~h}$, (b) $6 \mathrm{~h}$, (c) $12 \mathrm{~h}$, and (d) $18 \mathrm{~h}$.

the tendency for the thicker PVP-HDA film to undergo greater changes with increasing exposure time. The changes become more obvious in the first $12 \mathrm{~h}$. The line graphs are shown in Fig. S2.

Table 2 not only compares the changes of $C_{i}$ which are related to the thickness of PVP-HDA films, but also illustrates that wet environment can increase the $C_{\mathrm{i}}$, which is supposed to cause a decrease in $\mu$. In order to further explore the influence of humidity, the devices fabricated with three different thicknesses of PVP-HDA films were placed in air ambient with $40 \%$ relative humidity for $6 \mathrm{~h}$, and then compared with the initial devices. The transfer characteristic curves are shown in Fig. 3.

Fig. 3a-c show the devices with three different thicknesses of PVP-HDA films, while a clear difference in $V_{\text {th }}$ and $\mu$ can be observed in Fig. $3 \mathrm{~d}-\mathrm{f}$. Regardless of the thickness, the $\mu$ and $V_{\text {th }}$ of the devices change significantly after being explored in $40 \%$ humidity air conditions for $6 \mathrm{~h}$. Furthermore, the saturation currents of the devices rise noticeably for 650 and 375-nm-thick PVP-HDA films. The statistical data of $V_{\text {th }}$ for the three different devices are shown in Fig. $3 \mathrm{~d}-\mathrm{f}$. The average $V_{\text {th }}$ for the 650-nm-thick PVP-HDA film changes from -17.5 to $-11.8 \mathrm{~V}$. In the same situation, the average $V_{\text {th }}$ of the devices with 375 and 280-nm-thick PVP-HDA films change from -12.4 to $-9.3 \mathrm{~V}$ and -5.8 to $-4.6 \mathrm{~V}$, respectively. Fig. 3g-i show the statistical data of $\mu$ for the
Table 2 Change of capacitance of the PVP-HDA films with different thicknesses and exposure times

\begin{tabular}{cccc}
\hline \multirow{2}{*}{ Time (h) } & \multicolumn{3}{c}{$C_{\mathrm{i}}\left(10^{-8} \mathrm{~F} \mathrm{~cm}^{-2}\right)$} \\
\cline { 2 - 4 } & $650 \mathrm{~nm}$ & $375 \mathrm{~nm}$ & $280 \mathrm{~nm}$ \\
\hline 0 & 0.72 & 1.01 & 1.25 \\
6 & 0.81 & 1.10 & 1.31 \\
12 & 0.89 & 1.17 & 1.33 \\
18 & 0.91 & 1.22 & 1.32 \\
24 & 0.94 & 1.24 & 1.33 \\
\hline
\end{tabular}

three different devices. The devices with 375-nm-thick PVP-HDA have the highest average $\mu$, and the $\mu$ value changes from 8.1 to $11.5 \mathrm{~cm}^{2} \mathrm{~V}^{-1} \mathrm{~s}^{-1}$ (Fig. 3h). As shown in Fig. $3 g$, i, the average $\mu$ of the devices with 650 and 280-nm-thick PVP-HDA films change from 0.5 to $0.8 \mathrm{~cm}^{2} \mathrm{~V}^{-1} \mathrm{~s}^{-1}$ and 2.8 to $3.9 \mathrm{~cm}^{2} \mathrm{~V}^{-1} \mathrm{~s}^{-1}$, respectively. Furthermore, the original data for the three different PVP-HDA films were compared with the subtractive values, which were obtained via exposing the devices at $40 \%$ humidity air conditions for $6 \mathrm{~h}$ (shown in Fig. S3). Figs 2, 3 illustrate that the large improvement in saturation current results in a significant increase in $\mu$, especially in the first $6 \mathrm{~h}$.

In order to obtain more reliable changes of $\mu$, the factor $r$ was also introduced, as shown in Table S1. The corre- 

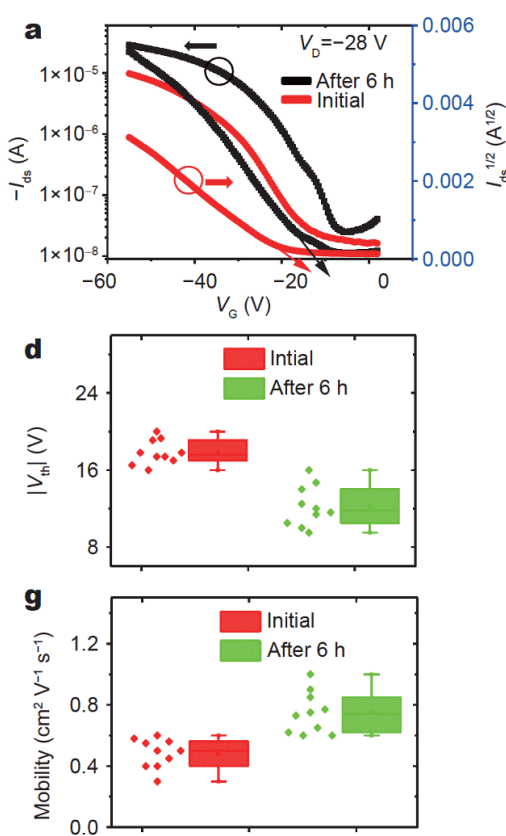

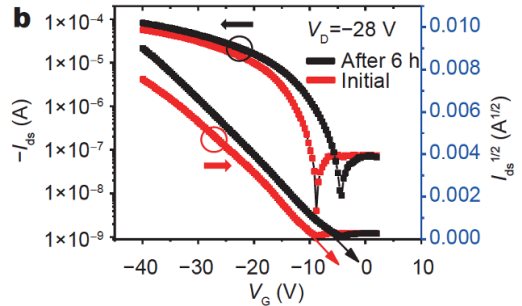

e

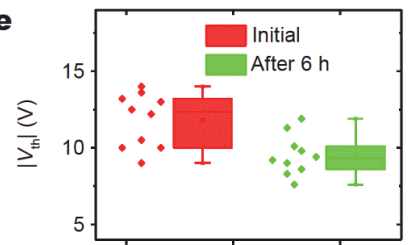

h

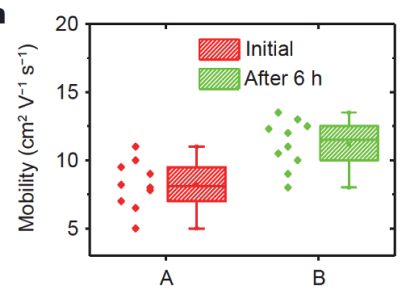

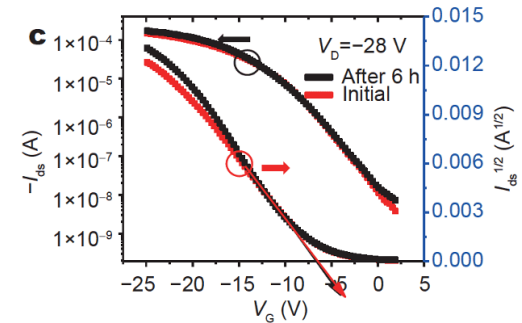

$\mathbf{f}$

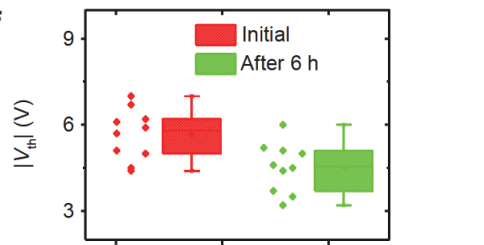

i



Figure 3 Typical characteristic curves of three different OTFTs and statistical data. The transfer characteristics of the OTFTs with (a) 650, (b) 375, and (c) 280-nm-thick PVP-HDA films as the gate dielectrics, respectively. The red curves represent the initial devices, while the black curves represent the devices after being placed in $40 \%$ humidity air conditions for $6 \mathrm{~h}$. (d-f) Statistical data of $V_{\text {th }}$ and (g-i) statistical data of $\mu$ for 650, 375, and 280-nm-thick PVP-HDA films, respectively.

sponding average $\mu$ value is shown in Table S2. Österbacka et al. $[35,36]$ demonstrated that PVP is a hygroscopic material and it can absorb water to cause ionic species motion. The ionic motion in the dielectric layer can increase the charge density at the interface between C8-BTBT and PVP-HDA layers, which can increase the saturation current. Another study has considered that PVP as a hydroxyl-rich material could absorb water and cause surface polarization, which also induces a higher saturation current [24]. In addition, hydroxyl groups in the dielectrics have been found to intensively trap electrons and completely eliminate electron transport in a variety of structurally disordered organic semiconductors [37], which may be beneficial to increasing the saturation current.

It is widely known that $C_{\mathrm{i}}$ is linked to both thickness and dielectric constant $(\varepsilon)$. The increase of $C_{\mathrm{i}}$ is basically caused by the change of $\varepsilon$, under the assumption that other parameters are unchanged. Thus, the PVP-HDA film absorbs the water, which causes the change of the dielectric constant, and $C_{\mathrm{i}}$ consequently increases. Previous study reported that [38]:

$V_{\mathrm{th}}=\frac{ \pm q n_{0} d}{C_{\mathrm{i}}}+V_{\mathrm{fb}}$.
Here, $q$ represents the elementary charge, $n_{0}$ represents the charge density, $d$ is the film thickness, $C_{\mathrm{i}}$ is the dielectric layer unit area capacitance, and $V_{\mathrm{fb}}$ is the flat band voltage, which accounts for any work-function difference between the semiconductor and the gate metal. On one hand, it has been proved that the devices using high-k dielectric materials tend to show a lower $\left|V_{\text {th }}\right|$ [39]. For PVP-HDA films, whether the rise of $C_{i}$ is caused by thickness or humidity exposure, it would cause a decline in $\left|V_{\text {th }}\right|$. The larger capacitance the films have, the smaller $V_{\text {th }}$ the devices perform. On the other hand, the AFM surface morphology images of the gate dielectric films with different thicknesses are shown in Fig. 4.

The root mean square (RMS) for all films increases after $12 \mathrm{~h}$ exposure in $40 \%$ humidity air. A rougher surface of the PVP-HDA layers would trap more free charges. The AFM height images of the C8-BTBT:PS films fabricated on 375-nm-thick PVP-HDA films are also shown in Fig. S4. There is a slight change in the RMS after $12 \mathrm{~h}$ exposure in $40 \%$ humidity air. This result demonstrates that the C8-BTBT:PS film is relatively stable in these conditions. Pernstich et al. [40] demonstrated that, with increasing negative gate voltage, more trap states were filled. In addition, the following equation can be 

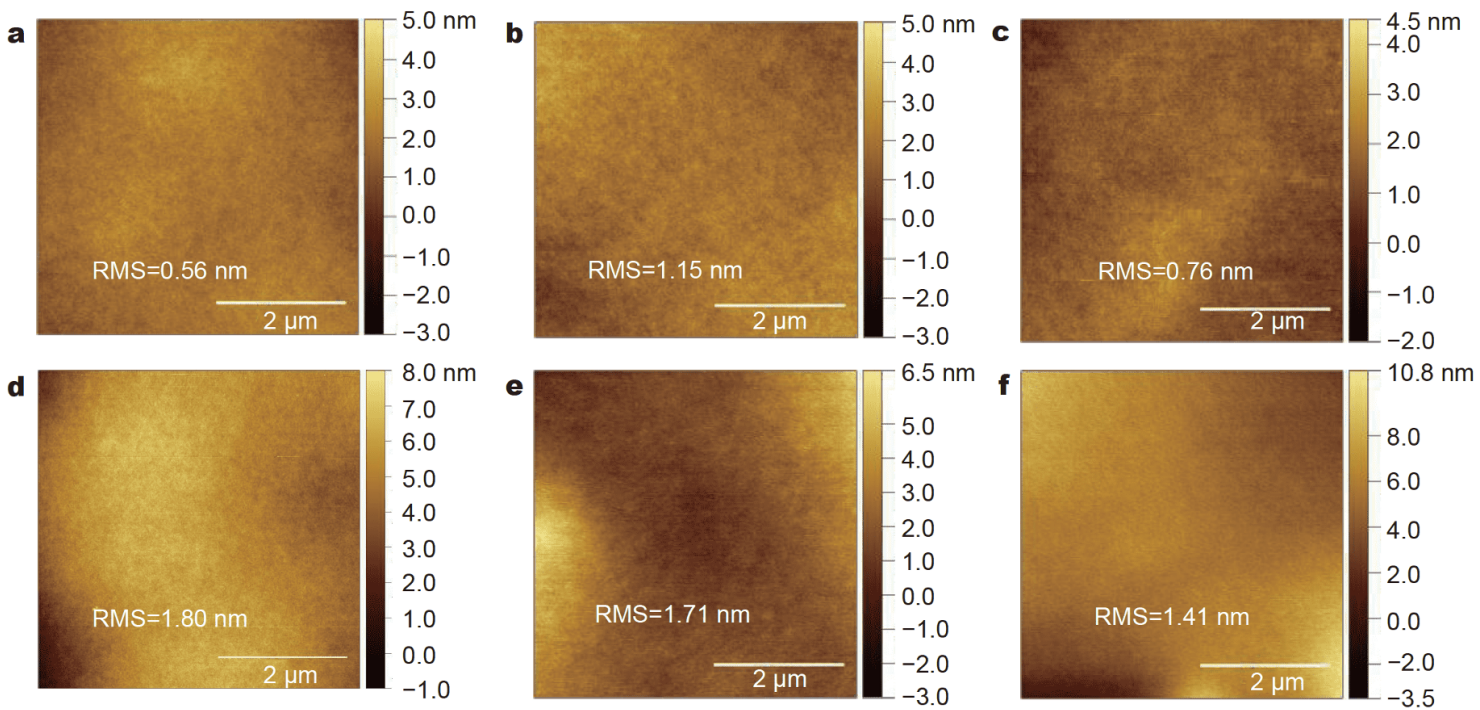

Figure 4 AFM height images of the PVP-HDA films with different thicknesses of (a) $650 \mathrm{~nm}$, (b) $375 \mathrm{~nm}$, and (c) $280 \mathrm{~nm}$. The corresponding images after $12 \mathrm{~h}$ exposure for the PVP-HDA films with the thicknesses of (d) $650 \mathrm{~nm}$, (e) $375 \mathrm{~nm}$, and (f) $280 \mathrm{~nm}$.

used to calculate $N_{\text {trap }}$ :

$N_{\text {trap }}=C_{\mathrm{i}}\left|V_{\text {tto }}\right| / e$,

where $V_{\text {tto }}=V_{\text {th }}-V_{\text {to }}, V_{\text {th }}$ means the threshold voltage, and $V_{\text {to }}$ represents the turn-on voltage, which is the gate voltage where the drain current starts to increase exponentially. It is possible to obtain $N_{\text {trap }}$ from Fig. 2a with $V_{\text {th }}=-9.5 \mathrm{~V}$ and $V_{\text {to }}=-8.5 \mathrm{~V}$, as well as from Fig. 2b with $V_{\text {th }}=-5.6 \mathrm{~V}$ and $V_{\text {to }}=-4.8 \mathrm{~V}$. Therefore, the $N_{\text {trap }}$ values for Fig. $2 \mathrm{a}, \mathrm{b}$ are $6.32 \times 10^{10} \mathrm{~cm}^{-2}$ and $5.50 \times 10^{10} \mathrm{~cm}^{-2}$, respectively.

In order to find out whether water molecules are absorbed by PVP-HDA, elemental analysis in crosssectional images is shown in Fig. 5. Since a better crosssection could be obtained with a rigid substrate, the devices were fabricated on glass substrates with $375-\mathrm{nm}$ -thick dielectric layer. Then the devices were exposed in
$40 \%$ humidity air for $12 \mathrm{~h}$ and compared with the original films. The cross-sectional images of the two devices can be clearly seen (Fig. 5a, b) and the change of the oxygen element is noticeable. The percentage of the oxygen element changes from $19.8 \%$ to $26.1 \%$, which strongly proves that the PVP-HDA dielectric layer absorbs some substances containing oxygen, as shown in Fig. 5c. Furthermore, the devices were tested after $40 \%$ humidity exposure for $6 \mathrm{~h}$ and stored in the glove box (where the water content is less than $0.1 \mathrm{ppm}$ ) for $24 \mathrm{~h}$. It is clear that the performance of the devices would drop, as shown in Fig. S5. The green curves indicate that, after $24 \mathrm{~h}$ drying, $V_{\text {th }}$ and $I_{\mathrm{ds}}$ are close to the initial values. The statistics of $V_{\text {th }}$ and saturated $I_{\text {ds }}$ are also shown in Fig. S5. In addition, $C_{\mathrm{i}}$ as an important parameter was also tested with the same method, and the statistic values are shown in Fig. S6. It is obvious that the performance of the devices
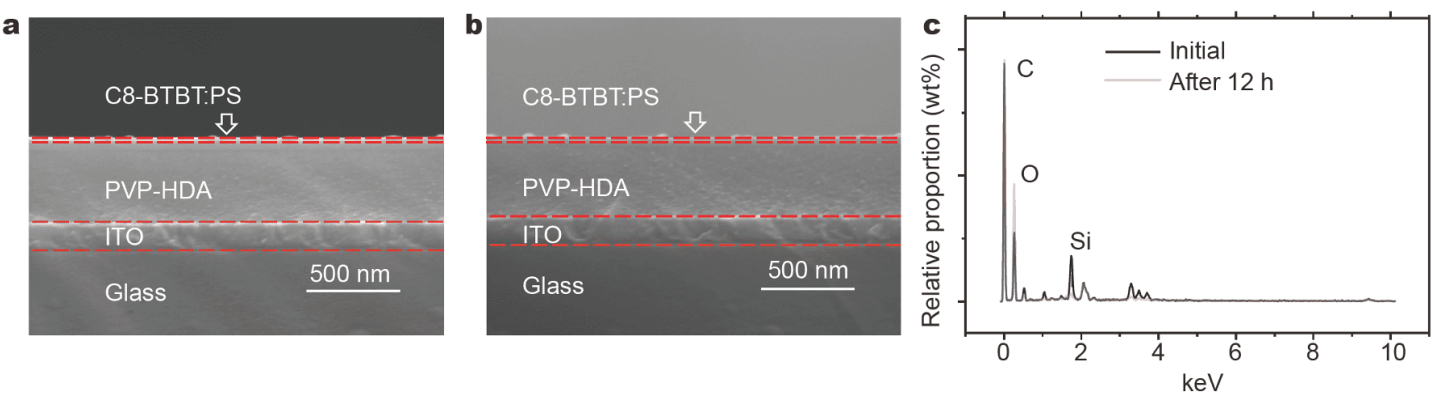

Figure 5 Section diagram of the devices fabricated with 375-nm-thick PVP-HDA film (a) initial and (b) after exposure in 40\% humidity air conditions for $12 \mathrm{~h}$. (c) Element content of the two different devices in (a) and (b). 
would recover after drying, including $V_{\text {th }}$, saturated $I_{\mathrm{ds}}$, and $C_{\mathrm{i}}$. The phenomenon illustrates that the changes of the devices can be eliminated after a period of drying and further proves the presence of absorbed water in the PVPHDA films.

The PVP-HDA films with three different thicknesses were exposed in air environment with humidity of $40 \%$ for $12 \mathrm{~h}$ and the evolution of the contact angles was compared, as shown in Fig. S7. The contact angle has an observable change, regardless of the thickness of the films. The change of contact angle of the PVP-HDA films with different thicknesses is slightly different. However, the film with a thickness of $650 \mathrm{~nm}$ has the most obvious change from $70.1^{\circ}$ to $68.1^{\circ}$ (Fig. S7a, b). The other films have slightly different contact angle, which is shown in Fig. S7c-f. The decrease of contact angle means the hydrophilicity of the films has been improved [41].

This also proves that the PVP-HDA films could be affected by wet environment and could absorb water from air. In Pernstich's study, it was demonstrated that a selfassembled monolayer (SAM) with a permanent electric dipole field inserted between the gate insulator and the pentacene would increase the band bending and enhance hole density in the channel [40]. Jung et al. [24] also demonstrated that, when the dielectric is porous, water molecules could be absorbed in the dielectric layer causing a greater amount of polarization in the dielectric. This effect can vastly overwhelm the bias stress and cause an increase in the drain current. It is believed that the parameters of the devices (including $V_{\mathrm{th}}, I_{\mathrm{ds}}$, and $\mu$ ) are changed due to both the adsorbed water and PVP-HDA films. On one hand, for the PVP-HDA films with same thickness, the rise of $C_{\mathrm{i}}$ with increasing exposure time would lead to a high carrier density and the decrease of the absolute value of $V_{\text {th }}$ [42]. On the other hand, even weak dopants can improve the device performance [43]. It is considered that the protons diffuse into the hygroscopic dielectric layer, resulting in doping of the semiconducting channel and increase of $I_{\mathrm{ds}}$ [44-46].

\section{CONCLUSIONS}

In conclusion, flexible C8-BTBT OTFT devices were fabricated with three different thicknesses of PVP-HDA films. It was proven that short-term air exposure for $6 \mathrm{~h}$ could improve the performance of the devices, including $\mu$ (the corrected average value changes from 3.2 to $5.1 \mathrm{~cm}^{2} \mathrm{~V}^{-1} \mathrm{~s}^{-1}$ ) and $V_{\text {th }}$ (the average value changes from -12.4 to $-9.3 \mathrm{~V}$ ). However, with the exposure time increasing beyond $6 \mathrm{~h}$, the insulation property and surface morphology of the PVP-HDA films were found to suffer some degradation and had a negative effect on $I_{\mathrm{on}} / I_{\text {off }}$ and $\mu$. Furthermore, the thicker PVP-HDA film has a more significant impact on the device performance, which is possibly due to the stronger water absorption. As demonstrated above, different from previous studies where the wet environment was shown to degrade the performance of OTFTs, in the present study our devices showed an enhanced electrical performances under a short-term air exposure. Therefore, it is envisaged that these results will provide a guiding role for the fabrication of highperformance OTFT devices in air environment.

\section{Received 27 February 2020; accepted 11 August 2020;}

published online 13 October 2020

1 Yuan Y, Giri G, Ayzner AL, et al. Ultra-high mobility transparent organic thin film transistors grown by an off-centre spin-coating method. Nat Commun, 2014, 5: 3005

2 Giri G, Verploegen E, Mannsfeld SCB, et al. Tuning charge transport in solution-sheared organic semiconductors using lattice strain. Nature, 2011, 480: 504-508

3 Duan S, Gao X, Wang Y, et al. Scalable fabrication of highly crystalline organic semiconductor thin film by channel-restricted screen printing toward the low-cost fabrication of high-performance transistor arrays. Adv Mater, 2019, 31: 1807975

4 Yang J, Yan D, Jones TS. Molecular template growth and its applications in organic electronics and optoelectronics. Chem Rev, 2015, 115: 5570-5603

5 Diao Y, Shaw L, Bao Z, et al. Morphology control strategies for solution-processed organic semiconductor thin films. Energy Environ Sci, 2014, 7: 2145-2159

6 Chen Y, Au J, Kazlas P, et al. Flexible active-matrix electronic ink display. Nature, 2003, 423: 136

7 Jiang C, Choi HW, Cheng X, et al. Printed subthreshold organic transistors operating at high gain and ultralow power. Science, 2019, 363: 719-723

8 Wang S, Xu J, Wang W, et al. Skin electronics from scalable fabrication of an intrinsically stretchable transistor array. Nature, 2018, 555: 83-88

9 Tong S, Sun J, Wang C, et al. High-performance broadband perovskite photodetectors based on $\mathrm{CH}_{3} \mathrm{NH}_{3} \mathrm{PbI}_{3} / \mathrm{C} 8 \mathrm{BTBT}$ heterojunction. Adv Electron Mater, 2017, 3: 1700058

10 Qian C, Kong L, Yang J, et al. Multi-gate organic neuron transistors for spatiotemporal information processing. Appl Phys Lett, 2017, 110: 083302

11 Kong L, Sun J, Qian C, et al. Long-term synaptic plasticity simulated in ionic liquid/polymer hybrid electrolyte gated organic transistors. Org Electron, 2017, 47: 126-132

12 Wei P, Li S, Li D, et al. Organic-semiconductor: Polymer-electret blends for high-performance transistors. Nano Res, 2018, 11: 5835-5848

13 Teixeira da Rocha C, Haase K, Zheng Y, et al. Solution coating of small molecule/polymer blends enabling ultralow voltage and high-mobility organic transistors. Adv Electron Mater, 2018, 4: 1800141

14 Zhou B, Zhou J, Chen Y, et al. Performance improvement of organic phototransistors by using polystyrene microspheres. Sci China Mater, 2018, 61: 737-744 
15 Gu PY, Wang Z, Liu G, et al. Synthesis, full characterization, and field effect transistor behavior of a stable pyrene-fused $\mathrm{N}$-heteroacene with twelve linearly annulated six-membered rings. Chem Mater, 2017, 29: 4172-4175

$16 \mathrm{Wu} \mathrm{X}, \mathrm{Du}$ R, Fang L, et al. Grain size adjustion in organic fieldeffect transistors for chemical sensing performance improvement. Sci China Mater, 2019, 62: 138-145

17 Qian C, Sun J, Zhang L, et al. Air-stable and high-performance organic field-effect transistors based on ordered, large-domain phthalocyanine copper thin film. Synth Met, 2015, 210: 336-341

18 Guo X, Ortiz RP, Zheng Y, et al. Thieno[3,4-c]pyrrole-4,6-dionebased polymer semiconductors: toward high-performance, airstable organic thin-film transistors. J Am Chem Soc, 2011, 133: 13685-13697

19 Lee WY, Oh JH, Suraru SL, et al. High-mobility air-stable solutionshear-processed $\mathrm{n}$-channel organic transistors based on corechlorinated naphthalene diimides. Adv Funct Mater, 2011, 21: 4173-4181

20 Kim SH, Yang H, Yang SY, et al. Effect of water in ambient air on hysteresis in pentacene field-effect transistors containing gate dielectrics coated with polymers with different functional groups. Org Electron, 2008, 9: 673-677

21 Zhang J, Jin J, $\mathrm{Xu} \mathrm{H}$, et al. Recent progress on organic donoracceptor complexes as active elements in organic field-effect transistors. J Mater Chem C, 2018, 6: 3485-3498

22 Liu D, Chu Y, Wu X, et al. Side-chain effect of organic semiconductors in OFET-based chemical sensors. Sci China Mater, 2017, 60: 977-984

23 Mai J, Tang N, He W, et al. Effects of ambient gases on the electrical performance of solution-processed C8-BTBT thin-film transistors. Nanoscale Res Lett, 2019, 14: 169

24 Jung T, Dodabalapur A, Wenz R, et al. Moisture induced surface polarization in a poly(4-vinyl phenol) dielectric in an organic thinfilm transistor. Appl Phys Lett, 2005, 87: 182109

25 Un HI, Cheng P, Lei T, et al. Charge-trapping-induced non-ideal behaviors in organic field-effect transistors. Adv Mater, 2018, 30: 1800017

26 Jurchescu OD, Baas J, Palstra TTM. Electronic transport properties of pentacene single crystals upon exposure to air. Appl Phys Lett, 2005, 87: 052102

27 Goldmann C, Gundlach DJ, Batlogg B. Evidence of water-related discrete trap state formation in pentacene single-crystal field-effect transistors. Appl Phys Lett, 2006, 88: 063501

28 Roberts ME, Queralto N, Mannsfeld SCB, et al. Cross-linked polymer gate dielectric films for low-voltage organic transistors. Chem Mater, 2009, 21: 2292-2299

29 Janneck R, Pilet N, Bommanaboyena SP, et al. Highly crystalline C8-BTBT thin-film transistors by lateral homo-epitaxial growth on printed templates. Adv Mater, 2017, 29: 1703864

30 Huang Y, Sun J, Zhang J, et al. Controllable thin-film morphology and structure for 2,7-dioctyl[1]benzothieno[3,2-b][1]benzothiophene (C8BTBT) based organic field-effect transistors. Org Electron, 2016, 36: 73-81

31 Xie P, Liu T, Sun J, et al. Solution-processed ultra-flexible C8BTBT organic thin-film transistors with the corrected mobility over $18 \mathrm{~cm}^{2} /(\mathrm{V}$ s). Sci Bull, 2020, 65: 791-795

32 Haase K, Teixeira da Rocha C, Hauenstein C, et al. High-mobility, solution-processed organic field-effect transistors from C8-BTBT: polystyrene blends. Adv Electron Mater, 2018, 4: 1800076

33 Choi HH, Cho K, Frisbie CD, et al. Critical assessment of charge mobility extraction in FETs. Nat Mater, 2018, 17: 2-7

34 He D, Wang Y, Huang Y, et al. High-performance black phosphorus field-effect transistors with long-term air stability. Nano Lett, 2019, 19: 331-337

35 Bäcklund TG, Sandberg HGO, Österbacka R, et al. Current modulation of a hygroscopic insulator organic field-effect transistor. Appl Phys Lett, 2004, 85: 3887-3889

36 Sandberg H, Bäcklund T, Österbacka R, et al. High-performance all-polymer transistor utilizing a hygroscopic insulator. Adv Mater, 2004, 16: 1112-1115

37 Jiang H, Huang Z, Xue G, et al. Electron transport at the interface of organic semiconductors and hydroxyl-containing dielectrics. J Mater Chem C, 2018, 6: 12001-12005

38 Egginger M, Bauer S, Schwödiauer R, et al. Current versus gate voltage hysteresis in organic field effect transistors. Monatsh Chem, 2009, 140: 735-750

39 Sun X, Di C, Liu Y. Engineering of the dielectric-semiconductor interface in organic field-effect transistors. J Mater Chem, 2010, 20: 2599-2611

40 Pernstich KP, Haas S, Oberhoff D, et al. Threshold voltage shift in organic field effect transistors by dipole monolayers on the gate insulator. J Appl Phys, 2004, 96: 6431-6438

41 Wei W, Yang C, Mai J, et al. High mobility solution-processed $\mathrm{C}_{8-}$ BTBT organic thin-film transistors via UV-ozone interface modification. J Mater Chem C, 2017, 5: 10652-10659

42 Fujimoto T, Matsushita MM, Awaga K. Ionic-liquid component dependence of carrier injection and mobility for electric-doublelayer organic thin-film transistors. J Phys Chem C, 2012, 116: 5240-5245

43 Wang Z, Zou Y, Chen W, et al. The role of weak molecular dopants in enhancing the performance of solution-processed organic field-effect transistors. Adv Electron Mater, 2019, 5: 1800547

44 Yambem SD, Burns S, Arthur JN, et al. A highly porous and conductive composite gate electrode for OTFT sensors. RSC Adv, 2019, 9: 7278-7284

45 Elkington D, Belcher WJ, Dastoor PC, et al. Detection of salivarange glucose concentrations using organic thin-film transistors. Appl Phys Lett, 2014, 105: 043303

46 Elkington D, Wasson M, Belcher W, et al. Printable organic thin film transistors for glucose detection incorporating inkjet-printing of the enzyme recognition element. Appl Phys Lett, 2015, 106: 263301

Acknowledgements This work was supported by the National Key Research and Development Program of China (2017YFA0206600) and the National Natural Science Foundation of China (51673214).

Author contributions Xie P and Liu T performed the experiments. Xie P, Liu T, Sun J and Yang J prepared the manuscript. Xie P, Sun J, He $P$, Dai G, Jiang J and Yang J participated in the discussion on experimental results. Yang J directed this project.

Conflict of interest The authors declare no conflict of interest.

Supplementary information online version of the paper. 


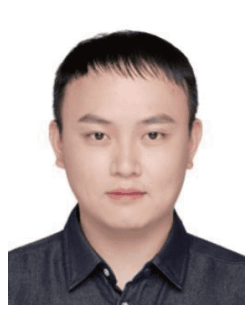

Pengshan Xie received his BSc degree from the Central South University in 2017. Currently, he is a graduate student at the Central South University. His research interests are focused on the functional layers of high-performance organic thin-film transistors.

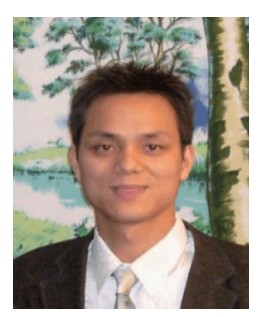

Junliang Yang received his $\mathrm{PhD}$ degree in 2008 from Changchun Institute of Applied Chemistry Chinese (CIAC), Chinese Academy of Sciences (CAS). He then joined Prof. Tim S. Jones's group at the University of Warwick. In April 2011, he moved to the University of Melbourne and the Commonwealth Scientific and Industrial Research Organisation (CSIRO). In 2012, he was appointed as a professor of the School of Physics and Electronics, Central South University. His research focuses on flexible and printed electronics, organic and perovskite solar cells.

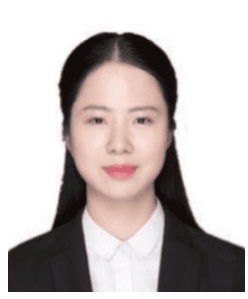

Tianjiao Liu received her BSc degree from $\mathrm{Hu}$ nan Normal University in 2016. She received her MSc degree from the Central South University in 2019. Her research interest focuses on high-performance organic thin-film transistors.

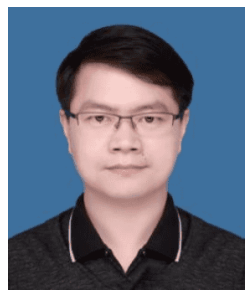

Jia Sun received his $\mathrm{PhD}$ degree from Hunan University in 2012. He was a postdoctoral researcher in the Central South University (20122014) and Sungkyunkwan University (20172018). In 2014, he joined the faculty of Central South University. Now he is a professor in the School of Physics and Electronics. His research interests focus on novel photoelectronic devices and neuromorphic devices.

\section{空气暴露对基于吸湿绝缘层的C8-BTBT有机薄膜 晶体管器件性能的影响}

谢朋杉, 刘天娇, 何培, 代国章, 蒋杰, 孙佳 ${ }^{*}$, 阳军亮 ${ }^{*}$

摘要 有机薄膜晶体管(OTFTs)通常对环境条件敏感, 在空气中暴 露时其性能往往会出现退化. 本文分析了基于聚(4-乙基苯酚)-4,4'(六氟异丙烯)二酞酸酐交联物绝缘层(PVP-HDA)的 2,7-二辛基 [1] 苯并噻吩 $[3,2-b][1]$ 苯并噻吩 (C8-BTBT) 柔性OTFTs 在 $40 \%$ 相对湿 度的空气环境中暴露不同时间对器件性能的影响. 研究发现, PVPHDA 绝缘层材料在短时间 $40 \%$ 相对湿度的空气下吸附空气中的水 分后, 器件的性能有所提升. 其中, 基于 $375 \mathrm{~nm}$ PVP-HAD绝缘层薄 膜的柔性OTFTs 在相对湿度为 $40 \%$ 的空气中放置6小时后, 校正后 的平均迁移率 $(\mu)$ 由 $3.2 \mathrm{~cm}^{2} \mathrm{~V}^{-1} \mathrm{~s}^{-1}$ 提高到 $5.1 \mathrm{~cm}^{2} \mathrm{~V}^{-1} \mathrm{~s}^{-1}$, 平均阈值 电压 $\left(V_{\mathrm{th}}\right)$ 由 $-12.4 \mathrm{~V}$ 降低到 $-9.3 \mathrm{~V}$, 开关比仍保持在 $10^{4}$. 结果表明, 此类OTFT器件有望能够在空气环境中进行大面积制备, 并展现出 良好的应用前景. 